\title{
Why do young adults living with HIV perform poorly on combined antiretroviral therapy (CART)? - a Zimbabwean perspective
}

\author{
Munyaradzi Madhombiro*,1, Raymond Cha², Joshua Sawyer ${ }^{2}$, Sarahmona Przybyla ${ }^{3}$, Gale \\ Burstein $^{4}$ \& Gene D Morse ${ }^{5}$ \\ ${ }^{1}$ University of Zimbabwe, College of Health Sciences, Department of Psychiatry, Parirenyatwa Group of Health Sciences, Mazowe \\ Street, Belgravia, Harare, Zimbabwe \\ ${ }^{2}$ University at Buffalo, The State University of New York, School of Pharmacy \& Pharmaceutical Sciences, Buffalo, NY 14414, USA \\ ${ }^{3}$ University at Buffalo, The State University of New York, School of Public Health, Buffalo, NY 14214, USA \\ ${ }^{4}$ Commissioner of Health Erie County, Health 95 Franklin St, Room 910, Buffalo, NY 14202, USA \\ ${ }^{5}$ University at Buffalo, The State University of New York, Pharmacy Practice (Medicine, Pediatrics), SUNY Global Health Institute, UB \\ Center for Integrated Global Biomedical Sciences, Drug Development Core, UB Clinical \& Translational Science Institute, \\ Translational Pharmacology Research Core, NYS Center of Excellence in Bioinformatics \& Life Sciences, University at Buffalo, 701 \\ Ellicott Street, Buffalo, NY 14203, 7168817464 \\ *Author for correspondence: munyarad@buffalo.edu
}

\section{"In order to achieve these goals, major obstacles, such as achieving optimal CART and preventing new infections, need to be adequately addressed"”}

First draft submitted: 12 February 2019; Accepted for publication: 18 February 2019; Published online: 29 April 2019

Keywords: antiretroviral • mental • neurological • perinatal HIV • young adults

Since the advent of antiretroviral therapy, there has been a significant decline in HIV incidence worldwide. However, less progress has been made with disease control and prevention in young adults (aged 19-25 years), as evidenced by higher infection incidence, poor virologic control and a disproportionally high mortality rate [1]. The transition from adolescence to young adulthood, between the ages of 15 and 24, is complicated by sociobehavioral and economic factors, alongside challenges with HIV status disclosure. Several comorbid medical conditions, including those related to mental health issues, neurological conditions and opportunistic infections (e.g., tuberculosis) may contribute to poor HIV treatment outcomes. Specifically, insufficient attention has been paid to comorbidities (Table 1) such as depression, anxiety, post-traumatic stress disorder (PTSD) and alcoholism and substance use disorders. Further treatment-related factors, such as newly-gained responsibility for self-care with antiretroviral medication, medication storage, management of adverse effects and transportation to clinical services may also impact health outcomes in this population. Additionally, the influence of neurocognitive disorders such as HIVassociated neurocognitive disorder (HAND), a recognized complication of chronic HIV infection, has not been adequately addressed in patients during this transition.

\section{Transitioning}

There are many clinical situations where adolescents and young adults must relocate to obtain adult care services when they transition services, for example, in diabetes care, congenital cardiac conditions and HIV care. This is particularly true in patients with complex chronic comorbidities. In many instances, this is difficult to do seamlessly and leads to a temporary breakdown or delay in care. While transition of care requires careful planning, the process is often haphazard, and the caregivers are often not prepared; some perinatally infected adolescents may already be doing poorly on treatment due to suboptimum adherence and unintended disclosure may exacerbate this situation. Limited efforts have been made to develop coordinated, collaborative and integrated transition programs [2], with no standardized protocol that optimally streamlines the transition for all patients and no systematic reviews giving specific recommendations for standard processes [3]. There is a critical need to develop well-planned, adolescent- 
Table 1. Factors requiring additional evaluation.

\begin{tabular}{ll} 
Psychosocial & $\begin{array}{l}\text { Poverty, homelessness, food insecurity, serostatus disclosure, relationships, } \\
\text { unemployment, discrimination and abuse/violence }\end{array}$ \\
\hline $\begin{array}{l}\text { Mental health } \\
\text { Neurological }\end{array}$ & $\begin{array}{l}\text { Depression, psychosis, anxiety, substance and alcohol use, PTSD, adjustment and } \\
\text { suicidality } \\
\text { HAND, neurodevelopmental and cognitive disorders }\end{array}$ \\
\hline Opportunistic infections & $\begin{array}{l}\text { Tuberculosis, toxoplasmosis, malignancies, cytomegalovirus, pneumonia and fungal } \\
\text { infections }\end{array}$ \\
\hline
\end{tabular}

focused transition frameworks involving both adult services providers, adolescent providers, families and adolescents themselves [2].

\section{Poverty}

Many young adults who have grown up living with HIV may never have been part of a family, as orphans before the combination antiretroviral therapy (CART) era. With the loss of parents through AIDS-related deaths, these young adults have been forced to play the role of young caregivers for each other and for siblings. Parental presence would support timely medication refill, but in their absence, poor adherence specific to delayed refill is often inevitable. The lack of parental support is associated with its own added stressors. Food insecurity, failure to attend school and unstable, under-resourced communities place this population at increased risk for sexual exploitation. While attempting to care for their siblings and friends, they may engage in transactional sex which augments transmission risks. Poverty also leads to an increased probability of intergenerational sex, where younger women engage in sexual relationships with older men who may be more likely to be infected or at higher risk of acquiring HIV infection. While transactional sex is illegal in Zimbabwe and associated with stigma, there is a lack of protections afforded to these involved women in Zimbabwe. These women are in constant conflict with law enforcement agents. Faced with homelessness, many youth become 'street people' and face frequent arrests by law enforcement agencies. These stresses have likely increased their vulnerability to mental neurological and substance (MNS) use disorders. Thus, adolescents and young adults face many psychosocial and physical difficulties which require urgent attention [4].

\section{Disclosure}

Research has shown that HIV disclosure in young adults is not adequately planned and sometimes ends in suicide, homicides, intimate partner violence and sexual assault [5]. Various societal and religious interpretations of HIV understand the disease as a punishment from god and witchcraft resulting in ostracism of the HIV infected from community activities. Many face unplanned and accidental disclosure either in school or as they transition to different care services. Consequently, some school children may have to conceal self-administration of medication to nonideal treatment times. This contributes to poor adherence and subsequent HIV treatment failure. While many young adults may be in sexual relationships and/or planning marriages, the issue of serostatus disclosure to potential partners is challenging. This may lead to mental health problems such as anxiety, depression, substance use and other adjustment problems.

\section{Mental health}

Inadequate attention has been given to underpinning comorbidities such as depression, anxiety, mood disorders, psychotic disorders, HIV-associated cognitive disorders, and substance use disorders such as tobacco and alcohol use [6-8]. Empirical research shows that untreated mental illness in adolescents and young adults is common and results from the inherent difficulties of diagnosis, lack of integration of mental health services with HIV care and the stigma associated with mental illness and its treatment [9]. For adolescents and young adults with perinatal HIV infection, mental health problems may be related to poorer virological control and difficulties such as loss of parental care or discrimination based on their HIV serostatus [10]. Public health and medical communities cannot ignore the multifactorial effect of other factors such as poor nutrition and substance abuse. A recent review suggests that many adolescents and young adults with vertically acquired HIV infection are predisposed to neurocognitive disorders and neurodevelopmental delays [11]. Due to the presence of mental illness, some 
young adults forget to take their medication altogether or do not take it on time, often resulting in virological failure.

\section{Depression}

Depression is a common comorbid condition among patients living with HIV or AIDS, affecting more than a third of PLWH $[12,13]$. Depression in young adults may be related to: life-long stresses as the perinatally infected individuals come to terms with their status, chronic illness and treatment, and discrimination and stigma. Depression is associated with cognitive challenges including forgetfulness and young adults often fail in achieving viral suppression. In the brain, HIV causes neuronal death, inflammatory processes and the derangement of cortisol release in the hypothalamus-pituitary-adrenal (HPA) axis, leading to an imbalance in neurotransmitter concentrations or activity [14]. The combination of psychosocial stressors, stigma, absence of sustained psychological support and effects of the virus on the brain, renders these young people at risk of poor adherence and consequently, poor treatment outcomes.

\section{Post-traumatic stress disorder}

PTSD, is common among young adults living with HIV or AIDS (YALWHA) and is often associated with use of psychoactive substances. History of trauma is associated with increased sexual risk taking and transmission of HIV [15]. Thus, there is a bidirectional relationship between HIV and PTSD [16]. PTSD is associated with specific brain changes particularly in the amygdala and the hippocampus [17]. Structural or functional changes in the amygdala, the integrative center for emotions and motivation, has been linked with PTSD. The amygdala is particularly primed for fear and associated reaction of hyperarousal, reliving the situation, dreaming and general anxiety features. As a reaction and self-treatment, many PTSD sufferers then consume alcohol and other psychoactive substances as a coping mechanism. PTSD may also be related to living with a life-altering illness such as HIV impacting future healthy sexual relationships, the trauma associated with one's HIV status and its complications and social rejection by family and society.

\section{Psychosis}

Psychosis results in anxiety, fear and enhanced stigma associated with mental illness. Psychosis may result from direct viral effect on the limbic system which includes the brainstem structures such as the ventral tegmentum area, nucleus accumbens, amygdala and hippocampus, and dopamine signaling. Dopamine function has been associated with HIV infection as the dopamine receptors are associated with HIV cofactors such as CCR5 [18]. Psychosis plays a role in HIV stigma and psychosis and may impact suboptimal adherence leading to poor treatment outcomes. Often, young adults present to hospitals with acute psychosis which is not adequately investigated in many low-resource settings. Thus, it is unclear whether it results from poor virological control or other brain-damaging processes.

\section{Alcohol}

Studies have consistently shown that PLWH have a higher prevalence of alcohol use than those noninfected [19]. Alcohol has been shown to affect HIV throughout the treatment continuum. Alcohol is linked to the acquisition of the infection through condomless sex and sexually transmitted infections. Furthermore, alcohol use is associated with both poor engagement in care, poor adherence to treatment and failure to achieve essential viral suppression. Alcohol is an immunosuppressant and may play a role in the inability of PLWH to successfully achieve viral suppression. Both the HIV virus itself and alcohol use can lead to brain atrophy, suggesting a role for alcohol in neuronal deaths. Long term use of alcohol causes cognitive impairment that may itself lead to the poor ability to take CART as prescribed.

\section{Other substances}

Use of psychoactive substances, such as marijuana, has been linked with increased rates of HIV infection as they can lead to unsafe sexual practices. Certain psychoactive substances such as psychostimulants that include cocaine and methamphetamines, are facilitators in HIV infection as they interact with dopamine receptors, which appear to be accessory to HIV co-factors CCR5 [20]. The effects of illicit substances will predictably be worse in YALWHA over other subpopulations since the brain is still developing. Opiate use is also associated with self-administered 
injections, and the use of contaminated needles is linked to transmission of HIV and Hepatitis C. This problem is magnified with the increase of injection drug use in Zimbabwe [18].

\section{HIV \& the CNS}

Evidence suggests that the HIV virus enters the brain early in the course of the infection [19,20]. The entry may be due the migration of T-lymphocytes, breach of the blood-brain barriers that allow direct entry by what has come to be known as the 'Trojan horse mechanism' [21]. Once in the brain, the virus invades microglial cells and resident macrophages, which express HIV binding proteins. Additionally, monocytes that migrate from the periphery can carry the virus into the brain [22]. Once in the brain tissue, monocytes transform to become macrophage and phagocytosis by macrophages results in increased virus accumulation in the macrophages and the brain [23]. Once resident in the brain, the virus enter the astrocytes some of whom express the chemokine CCR5 and also the virus enters the astrocytes through cell-to-cell contact [24,25]. This leads to further damage of brain architecture including the blood-brain barrier and disturb neuronal metabolism where astrocytes are involved and once damaged, the overall function of the neurons is compromised that including oxidative stress and apoptosis [26].

Recent studies suggest that mitochondria is an integral part of the brain damage process in HANDs [27]. Direct viral effects on the brain tissue and inflammatory processes that ensue following the viral entry in the brain, may cause brain function derangement. Other cell lines affected include the myelin producing cells, the oligodendrocytes, where a loss of white matter is observed. The HIV virus in the brain leads to breakdown of the brain architecture including damage to the blood-brain barrier, neuronal damage due to inflammation, increased immune response and development of the HIV virus tat proteins. There is no evidence that these young adults would have special protective features from the cognitive impairment.

\section{HIV-associated neurocognitive disorders}

Although HAND has been reported in adults in high-income regions, it is not well characterized in young adults and has not been adequately studied in low resource settings. This condition has three categorical clades. Asymptomatic neurocognitive impairment (ANI), as the name implies, shows presence of disturbance in neuropsychological effects with no symptoms but with one standard deviation on the neuropsychological battery [28]. The second class is the minor neurocognitive impairment (MND) and is characterized by presence of two standard deviations on the neuropsychological tests. The extreme form is HIV-associated dementia (HAD). Incidence of HIV-associated dementia is increasing in elderly HIV populations but has not been characterized in young adults. These are serious conditions and may affect young adults. Although there has been a suggestion that HIV-1 clade C (seen in Zimbabwe and other African countries) does not cause HAND, increasing evidence is finding that this may not be accurate $[29,30]$. Thus, it is critical that these effects on the brain be further evaluated as their contribution to the observed high mortality in YALWH cannot be ruled-out.

\section{Future perspective}

HIV in young Zimbabwean adults remains a formidable challenge. As we struggle with morbidity and mortality among perinatally infected young adults, we must not forget the increasing number of young people who are acquiring the infection behaviorally. Sexual relationships between the perinatally infected and the HIV-negative population and intergenerational sex between young girls and elderly men contribute to incident cases in Zimbabwe. Many young adult females submit to transactional sex and reports show that transactional sex workers have $56 \%$ seropositivity in Zimbabwe [31]. Finally, the current HIV treatment regimens aim to achieve viral suppression, but the new frontier is to eradicate the virus and optimize prevention using both social and biomedical models such as pre-exposure prophylaxis (PrEP). While PrEP has become widely available in many developed nations, it is important for countries that still have a huge burden of HIV such as Zimbabwe, to embrace and disseminate this intervention. There is ongoing work in attempts to improve adherence and viral suppression by long-acting CART. Leveraging wide-reaching communication approaches may enhance treatment outcomes by use of shortmessage services, e-health smartphone applications, electronic record data processing and other bioinformatics platforms. 


\section{Research priorities}

- Develop a seamless transition process for HIV care for adolescents who are changing services;

- Establish resilience and establish a safety net for young adults in the face of psychosocial challenges faced by children born with HIV;

- Standardize and improve identification and management of mental health problems in young adults with HIV;

- Optimize diagnosis and treatment of neurocognitive disorders in young adults with HIV;

- Enhance interventions that promote adherence to CART;

- Improve availability of long acting CART to improve adherence in young adults;

- Leverage on information communication technologies to promote healthy choices in young adults with HIV.

\section{Challenges}

In order to achieve these goals, major obstacles, such as achieving optimal CART and preventing new infections, need to be adequately addressed. After investing sufficient resources in prevention and treatment, the need for advanced biomedical therapies that prevent or eliminate HIV has taken center stage. Justifiably, more resources are being devoted into this research, including pre- and post-exposure prophylaxis interventions. Widespread recognition that mental disorders increase vulnerability to HIV infection is imperative in moving towards achieving HIV elimination. The significance of sanctuary sites and reservoirs, including the brain, requires additional exploration. Although there is an increasing body of work on the disposition of drugs in the brain, there are significant gaps in the knowledge that need to be resolved before these concepts can be applied generally to YALWH.

\section{Conclusion}

In order to improve treatment outcomes with reductions in mortality in YALWHA, it is imperative that all the barriers to successful HIV treatment be adequately and appropriately addressed. As a country with a high HIV burden, Zimbabwe needs to train practitioners to deliver evidence-based HIV care, researchers who prioritize achieving optimal HIV outcomes and increase funding in HIV care including for developing evidence-based improved transitions for young adults to adult care. Poverty and general lack of resources faced by young adults must be addressed through increasing opportunities that are not necessarily based on their academic achievements but other technical and social vocations. Integrated social services that focus on this subpopulation must be explored. Developing mental health improvements will be key since these conditions are modifiable. Substantial enhancements are needed to address alcohol and substance use given its extensive influence in young adults. Neurocognitive disorders related to HIV infection must be better identified and addressed. Cultural and social barriers to engagement and linkage to HIV care including stigma related to religion and witchcraft needs to be dismantled. Disclosure of HIV status need to be vigilantly managed to facilitate access to continuous treatment for young adults. New infections must be prevented by expanding application of PrEP, Post-exposure prophylaxis (PEP), safe-sex practices and HIV testing particularly for young adults. Research into long-acting CART needs to be further explored.

\section{Summary points}

- Young people with perinatally acquired HIV perform poorly on treatment outcomes.

- Challenges include discontinuous transitioning care, poverty, disclosure, mental health issues, cognitive impairment and poor adherence.

- Interventions are needed urgently to reduce loss of life, new infections and associated morbidities in young adults. 


\section{References}

1. Judd A, Lodwick R, Noguera-Julian A et al. Higher rates of triple-class virological failure in perinatally HIV-infected teenagers compared with heterosexually infected young adults in Europe. HIV Med. 18(3), 171-180 (2017).

2. Lee S, Hazra R. Achieving 90-90-90 in paediatric HIV: adolescence as the touchstone for transition success. J. Int. AIDS Soc. 18(Suppl. 6), 20257 (2015).

3. Machado DM, Succi RC, Turato ER. Transitioning adolescents living with HIV/AIDS to adult-oriented health care: an emerging challenge. J. Pediatr. 86(6), 465-472 (2010).

4. Vreeman RC, Scanlon ML, McHenry MS et al. The physical and psychological effects of HIV infection and its treatment on perinatally HIV-infected children. J. Int. AIDS Soc. 18(Suppl. 6), 20258 (2015).

5. Arrive E, Dicko F, Amghar $\mathrm{H}$ et al. HIV status disclosure and retention in care in HIV-infected adolescents on antiretroviral therapy (ART) in West Africa. PLoS ONE 7(3), e33690 (2012).

6. Gamarel KE, Brown L, Kahler CW et al. Prevalence and correlates of substance use among youth living with HIV in clinical settings. Drug Alcohol Depend. 169, 11-18 (2016).

7. Maskew M, Fox MP, Evans D et al. Insights into adherence among a cohort of adolescents aged 12-20years in South Africa: reported barriers to antiretroviral treatment. AIDS Res. Treat. 2016, 1-12 (2016).

8. Willis N, Mavhu W, Wogrin C et al. Understanding the experience and manifestation of depression in adolescents living with HIV in Harare, Zimbabwe. PLoS ONE 13(1), e0190423 (2018).

9. Vreeman RC, McCoy BM, Lee S. Mental health challenges among adolescents living with HIV. J. Int. AIDS Soc. 20(Suppl. 3), 21497 (2017).

10. Lowenthal ED, Bakeera-Kitaka S, Marukutira T et al. Perinatally acquired HIV infection in adolescents from sub-Saharan Africa: a review of emerging challenges. Lancet Infect. Dis. 14(7), 627-639 (2014).

11. Laughton B, Cornell M, Boivin $\mathrm{M}$ et al. Neurodevelopment in perinatally HIV-infected children: a concern for adolescence. J. Int. AIDS Soc. 16, 18603 (2013).

12. Ayano G, Solomon M, Abraha M. A systematic review and meta-analysis of epidemiology of depression in people living with HIV in east Africa. BMC Psychiatry 18(1), 254 (2018).

13. Bigna JJ, Tounouga DN, Kenne AM et al. Epidemiology of depressive disorders in people living with HIV in Africa: a systematic review and meta-analysis: Burden of depression in HIV in Africa. Gen. Hosp. Psychiatry 57, 13-22 (2018).

14. Barroso J, Burrage J, Carlson J et al. Salivary cortisol values in HIV-positive people. J. Assoc. Nurses AIDS Care 17(3), 29-36 (2006).

15. Richter L, Makusha T, Komarek A et al. Exploring the impact of childhood abuse on HIV social and attitudinal factors among adults with and without this history in sub-saharan africa: findings from NIMH project accept (HPTN 043). AIDS Behav. 20(4), 737-745 (2016).

16. Rowe C, Santos GM, McFarland W et al. Prevalence and correlates of substance use among trans female youth ages 16-24 years in the San Francisco Bay Area. Drug Alcohol Depend. 147, 160-166 (2015).

17. Ahmed-Leitao F, Spies G, van den Heuvel L et al. Hippocampal and amygdala volumes in adults with posttraumatic stress disorder secondary to childhood abuse or maltreatment: a systematic review. Psychiatry Res. Neuroimaging 256, 33-43 (2016).

18. Zimbabwe Church, Health Ministry Launches Anti-Drug

Campaign (2019). www.voanews.com/a/zimbabwe-youth-substance-abuse/4735119.html

- Alcohol and substance use is a rising problem in all of Africa, andalso in Zimbabwe, and it has become one of our top three diagnoses.

19. Georgsson G. Neuropathologic aspects of lentiviral infections. Ann. NY Acad. Sci. 724, 50-67 (1994).

20. Persidsky Y, Poluektova L. Immune privilege and HIV-1 persistence in the CNS. Immunol. Rev. 213, 180-194 (2006).

21. Hazleton JE, Berman JW, Eugenin EA. Novel mechanisms of central nervous system damage in HIV infection. HIV AIDS 2, 39-49 (2010).

22. Williams DW, Veenstra M, Gaskill PJ et al. Monocytes mediate HIV neuropathogenesis: mechanisms that contribute to HIV associated neurocognitive disorders. Curr. HIV Res. 12(2), 85-96 (2014).

23. Lamers SL, Fogel GB, Nolan DJ et al. HIV-associated neuropathogenesis: a systems biology perspective for modeling and therapy. Biosystems 119, 53-61 (2014).

24. Brack-Werner R, Bell JE. Replication of HIV-1 in human astrocytes. NeuroAids 2(8), 1-22 (1999).

25. Li GH, Henderson L, Nath A. Astrocytes as an HIV reservoir: mechanism of HIV infection. Curr. HIV Res. 14(5), 373-381 (2016).

26. Eugenin EA, Clements JE, Zink MC et al. Human immunodeficiency virus infection of human astrocytes disrupts blood-brain barrier integrity by a gap junction-dependent mechanism. J. Neurosci. 31(26), 9456-9465 (2011).

27. Yu F, Hao Y, Zhao $\mathrm{H}$ et al. Distinct mitochondrial disturbance in $\mathrm{CD} 4+\mathrm{T}$ and $\mathrm{CD} 8+\mathrm{T}$ cells from hiv-infected patients. J. Acquir. Immune Defic. Syndr. 74(2), 206-212 (2017).

28. Saylor D, Dickens AM, Sacktor N et al. HIV-associated neurocognitive disorder - pathogenesis and prospects for treatment. Nat. Rev. Neurol. 12(5), 309 (2016). 
29. Joska JA, Fincham DS, Stein DJ et al. Clinical correlates of HIV-associated neurocognitive disorders in South Africa. AIDS Behav. 14(2), 371-378 (2010).

30. Ortega M, Heaps JM, Joska J et al. HIV clades B and C are associated with reduced brain volumetrics. J. Neurovirol. 19(5), 479-487 (2013).

31. UNAIDS 'AIDSinfo' (2019). www.unaids.org/en/resources/fact-sheet 
